\title{
Measuring the Impact of Agriculture Programs on Diets and Nutrition
}

Jef L Leroy, Marie Ruel and Deanna Olney, International Food Policy Research Institute

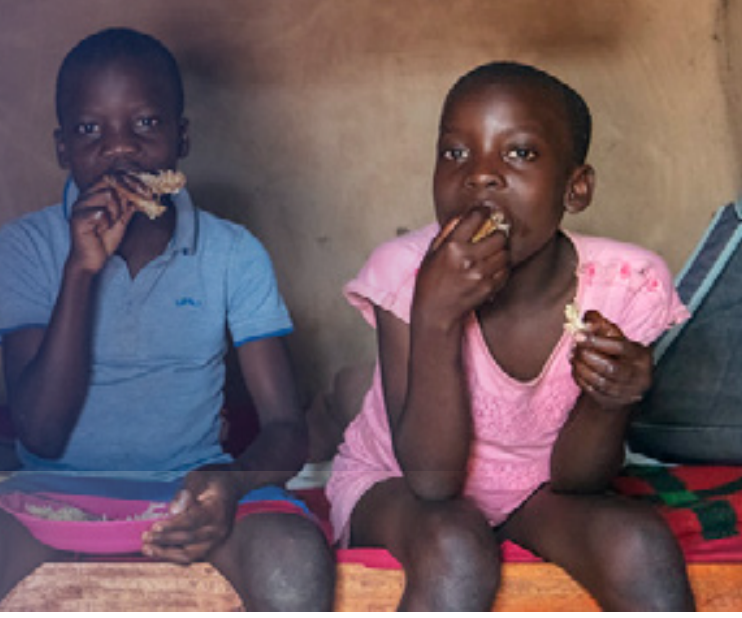

Agriculture holds tremendous potential to improve nutrition. Traditionally, agriculture investments focused on producing enough food to allow people to meet their caloric needs and on generating employment and income. In the last decade, the understanding of how agriculture can contribute to nutrition has shifted from the implicit assumption that increased productivity and income would automatically improve nutrition to the acknowledgement that explicit nutrition goals and actions are needed to improve nutritional outcomes (1-4). This has led to increased commitments and investments in nutrition-sensitive agriculture programs and accompanying research to study these programs' impact on nutrition outcomes. Guidance on how to make agriculture more nutrition-sensitive was also developed and included recommendations to target the first 1,000 days of a child's life (from conception to 2 years of age) and to focus on reducing stunting (5-7). These developments coincided with the global commitment to achieve the World Health Assembly target of reducing child stunting by 40 percent by 2025 (8).

\section{How Agriculture Programs Can Improve Nutrition: The Key Role of Diets}

Agriculture's primary role in improving nutrition is to increase access to nutritious foods and healthy diets, through several possible pathways (9). Increased agricultural production can improve diets through consumption from own production and through increased income from selling crops. Gender-sensitive agriculture programs, especially those directly targeting women, can increase women's decision-making power around using household resources to purchase nutritious foods. Using the additional income to increase the use of health and nutrition services can also improve child nutritional status. In addition, agricultural policies can impact food prices and increase or decrease the affordability of food. Agriculture can also have

\section{Key Messages}

- Agriculture programs can increase household access to nutritious foods and contribute to improving the diets of all household members. Greater impacts are found when strong health and nutrition behavior change communication (BCC) and women's empowerment activities are included.

- Assessing the impact of agriculture programs on nutrition should focus on measuring individual dietary intake and adequacy.

- Changes in micronutrient status, anemia, and overweight and obesity can also be used to measure nutrition impacts when interventions are designed to specifically address these outcomes.

- We do not recommend that agriculture programs use child stunting as a primary indicator of success.

unintended negative effects on women's time availability and their ability to care for young children, and on exposure to agriculture-associated diseases, chemicals, and toxins.

\section{Evidence of Agriculture Programs' Impact on Diet and Nutrition Outcomes}

A recent review summarized the diet and nutrition impacts of a range of agriculture programs implemented in the last decade, including studies on biofortification of staple crops, homestead food production, small livestock and dairy production, nutritionsensitive value chains, and irrigation (10). All programs aimed at increasing production diversity and household consumption of nutritious foods and succeeded in doing so. Where studied, positive impacts were also reported on maternal and child dietary 
diversity, anemia, vitamin A intake and status, and on maternal nutrition knowledge and practices. Beneficial effects on child diarrhea and illness, maternal underweight, child wasting, and women's empowerment were also reported. Greater impacts were found for programs that included strong health and nutrition behavior change communication (BCC) and women's empowerment activities. The only program with a positive impact on child stunting included these components plus a strong WASH intervention and/or the distribution of micronutrient supplements to children (Olney et al. unpublished results).

These findings show that although agriculture can reduce child stunting when coupled with lipid-based nutrient supplements and WASH interventions, its most consistent impacts have been on enhancing household and individual consumption of nutritious foods and diverse diets. Agriculture programs should therefore use improvements in diets as their main measure of success, rather than child stunting. There are two additional reasons for shifting the focus of agriculture interventions' impacts on nutrition to diets rather than child stunting. First, preventing child stunting entails much more than ensuring access to nutritious foods and feeding children adequate diets; it requires improving maternal nutrition, health, and water and sanitation knowledge and practices, empowering caregivers to adopt optimal feeding and caregiving practices, and

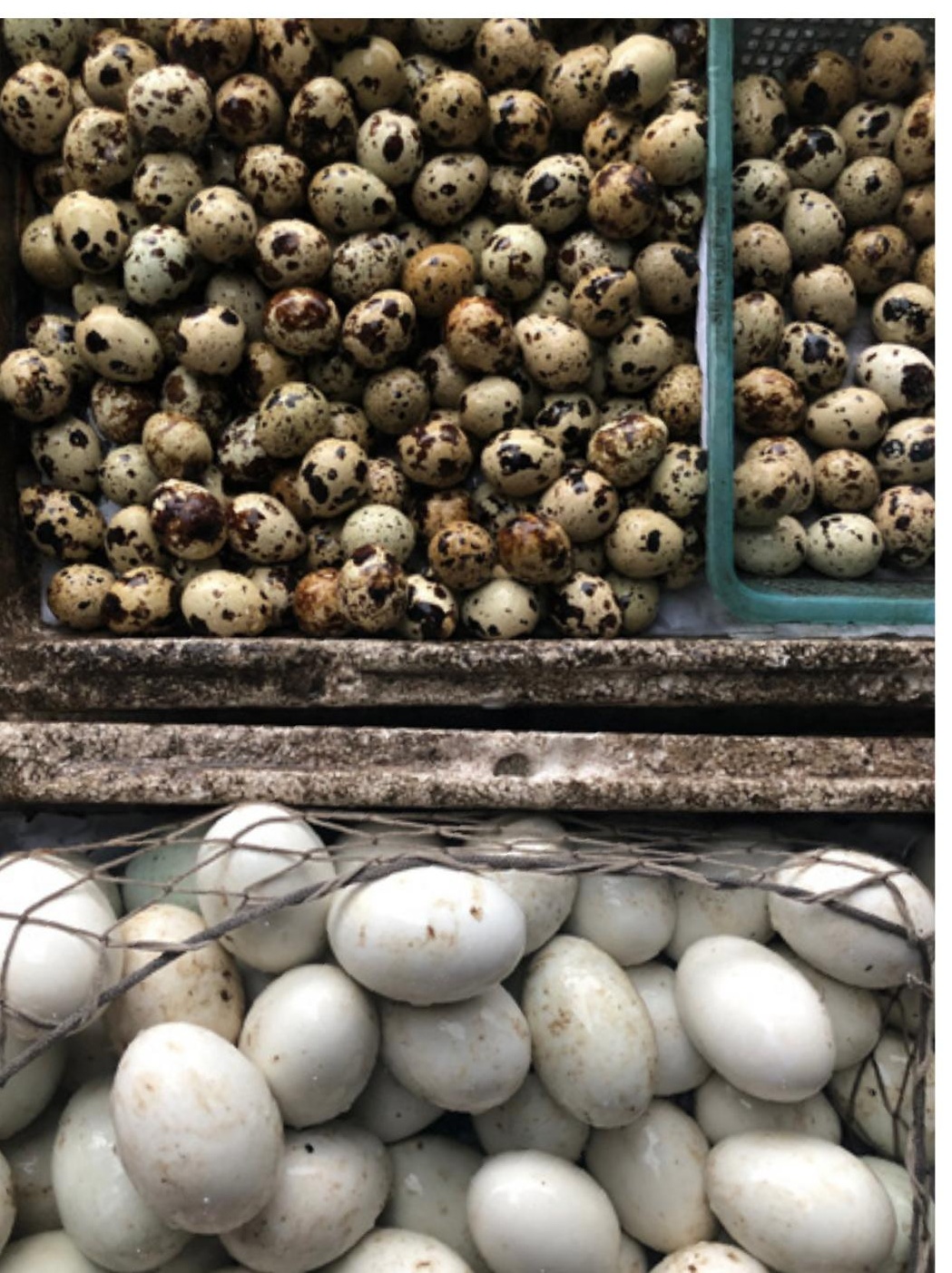

promoting the use of health services to prevent and control infections and other childhood illnesses (11). Also, young children and women during pregnancy and lactation have higher nutrient requirements than other household members and cannot always rely solely on the family diet, especially in poor resource-constrained settings. They often require specially formulated micronutrient supplements or fortified foods either provided by the health sector or purchased. Second, a recent review of what stunting ${ }^{1}$ really means shows it should not be used as a primary outcome to assess the impact of development programs in general (12). The widespread focus on stunting is based on the common misperception that being stunted in early childhood causes a variety of poor outcomes later in life, including delayed cognitive development, poor school performance, reduced productivity and earnings in adulthood, and a higher incidence of obesity and diet-related noncommunicable diseases. The review shows that stunting does not cause these outcomes, however, and therefore addressing stunting will not automatically lead to improvements in other development outcomes.

\section{Measuring the Impact of Agriculture Programs on Diets}

Unhealthy diets are at the root of all forms of malnutrition. Traditional cereal-based diets with low nutrient-density and poor micronutrient bioavailability are associated with micronutrient deficiencies and poor nutritional status. Dietary patterns in lowand middle-income countries (LMIC) are rapidly shifting, however, and diets are becoming too high in fat (often saturated fat), sugar, and salt, and increasingly include ultra-processed foods, while the intake of fruits and vegetables remains inadequate. These changes have contributed to the coexistence of undernutrition and micronutrient deficiencies with overweight, obesity, and diet-related noncommunicable diseases (13).

Several methods exist for dietary assessment. The multi-pass 24-hour recall is a commonly used method to measure diets and nutrient intakes in LMIC and is used to assess the quantity of all foods consumed by an individual during the 24 hours prior to the interview (14). Repeated 24-hour recalls on a sub-sample of a population are required to estimate the probability of adequacy of intake of energy and different nutrients. The food frequency (FFQ) approach uses a longer recall period, usually 7 to 30 days, and gathers information on the frequency the individual consumed foods from a pre-defined list during the recall period. The longer recall period allows for capturing

\footnotetext{
1 Stunting and linear growth retardation are not synonyms (12). Linear growth retardation refers to the failure to reach one's linear growth potential and implies children are too short for their age but does not imply they are stunted. Stunting is defined as having a height-for-age z-score (HAZ) below -2SD. Children who are stunted are a subset of those with linear growth retardation. For simplicity, we refer to both as "stunting" in this brief.
} 
foods not consumed every day but may introduce recall biases. The semi-quantitative version of FFQ uses portion sizes to collect information on quantity of food consumed (15). In combination with the appropriate food composition table, 24-hour recall and semi-quantitative $\mathrm{FFQ}^{2}$ data can be used to quantify intake of energy, individual nutrients, and fiber. Using 24-hour recall and semi-quantitative FFQ requires skilled interviewers and is time-consuming and costly, which can limit usefulness for large surveys.

Several composite measures of dietary quality have also been developed to simultaneously capture different dimensions of healthy and nutritious diets, including dietary adequacy (i.e., meeting nutrient requirements), diversity and proportionality (i.e., adherence to national dietary guidelines and recommended intakes for different food groups) and moderation (i.e. avoiding excess intake of select nutrients or food components) (16-18). Examples include the Diet Quality Index International (19), the Healthy Eating Index (20), and the Alternate Healthy Eating Index (21). These have mostly been validated in highincome countries, however, and would need to be validated and adapted for use in LMIC (17).

Dietary diversity (DD) indicators were developed largely to address the need for simpler indicators for use in large surveys to track progress and to measure program impacts in LMIC. DD indicators were validated for their performance in predicting the mean probability of micronutrient adequacy in children 6-23 months of age (22) and in women of reproductive age (23), respectively. These indicators provide a rough estimate of the probability of micronutrient adequacy based on the diversity of the diet and should be used only in contexts where DD is a known constraint to healthy diets. In countries where diets are diverse, but quantities of each food/food group are inadequate, DD indicators will fail to identify potentially serious dietary deficiencies. Another limitation is that DD indicators cannot capture excess consumption of energy, fats, sugars, salt, and ultra-processed foods, which is increasingly prominent in diets around the world and increases the risk of overweight and obesity and is associated with poor micronutrient intake $(23,24)$.

Challenges related to the complexity and cost of quantitative dietary assessment methods and the lack of simple, yet accurate, composite indicators of dietary quality are currently being addressed. The International Dietary Data Expansion Project (INDDEX) is developing an electronic approach to stream-

line and reduce the cost and time to conduct 24-hour recalls in LMIC and promote the timely use of decision-making information. The Intake Center for Dietary Assessment is developing and validating simple metrics and tools to track progress in achieving healthy diets in LMIC. Finally, there is a growing focus on the sustainability of diets. The WISH Index (under development by $\mathrm{A} 4 \mathrm{NH}$, led by Wageningen University) will capture how healthy diets are as well as how sustainable they are (Inge Brouwer, personal communication).

\section{Measuring the Impacts of Agriculture Programs on Diet-related Nutritional Status Outcomes}

Measuring the impacts of agriculture on diet-related nutritional status outcomes such as anemia, micronutrient status, and the prevalence of overweight and obesity can be useful but should only be included when interventions are designed to specifically address these outcomes.

There is some evidence that agriculture programs can reduce anemia (10). Given anemia's multifactorial causes, impacts could occur from improvements in micronutrient intake (e.g., iron, folate, vitamin B-12, vitamin C), from increased utilization of health services, and from reductions in infections. Impacts on anemia could also result from agriculture programs that reduce exposure to vector-borne diseases such as malaria or schistosomiasis. In areas where malaria is prevalent, assessment of malaria infection using rapid diagnostic tests (RDT) may be considered to interpret changes in anemia prevalence. Anemia and malaria infection can be assessed through a finger-prick blood sample and analyzed immediately, thus not requiring a cold chain, which is often a limiting factor in assessing other biomarkers.

Micronutrient status can be improved through biofortification and agriculture programs that promote the production and consumption of fruits, vegetables, fish, small animals, and eggs. For these types of programs, we recommend assessing impacts on the targeted nutrients (e.g., vitamin A, iron, and/or zinc). Recent developments in testing have made it possible to assess vitamin A and iron status through finger-prick samples. These assessments, however, still require specialized training, a cold chain, and laboratory processing and analysis capacity. Other micronutrient outcomes that may be relevant for agriculture programs include vitamin D, vitamin B-12, and folate status, but all these measures require venous blood samples, a cold chain, and laboratory processing and analysis, making them more challenging for field conditions in many LMIC.

Lastly, as agriculture programs transition into broader food systems development, it is increasingly important to assess exposure to the rapidly changing food environments in LMIC characterized by the modernization of the food retail sector. By increasing access to - and affordability of - nutritious foods and by providing nutrition BCC on healthy diets and lifestyles, 
agriculture programs may reduce the risk of overweight and obesity by keeping populations from consuming cheap, widely available, and aggressively promoted ultra-processed foods, snacks, and beverages. On the other hand, rising incomes from agricultural development programs may lead to negative dietary changes that may be difficult to counteract even with nutrition counseling and result in rapid rises in overweight and obesity as observed in many rural areas of LMIC (13). For these reasons, we recommend assessing the impact of agriculture programs on overweight and obesity in contexts where rapid modernization of food systems and environments is occurring.

\section{Outlook and Research Priorities}

Agriculture programs can increase household access to nutritious foods and contribute to improving the diets of all household members. In most resource-constrained environments, however, ensuring micronutrient adequacy of children under two and of pregnant and lactating women will require the additional provision of specially formulated micronutrient supplements or fortified foods. As rapidly changing food systems transform diets in both urban and rural populations, agriculture programs coupled with well designed and implemented BCC focused on healthy diets and lifestyles could protect poor households from food insecurity and ensure their stable access to - and consumption of - nutritious diets.

Quantifying the impacts of agriculture programs on dietary intake and adequacy remains challenging. Dietary diversity indicators offer a field-friendly approach to qualitatively assess diet quality, but do not allow for the identification of specific nutrient deficiencies or excess consumption of energy, saturated fat, sugar, or salt leading to overweight and obesity and diet-related noncommunicable diseases. Encouraging progress is being made, however, to develop new assessment methods; while some aim to simplify and increase efficiency of diet quality measurements, others are being developed to capture additional dietary aspects such as sustainability, which is particularly relevant from a food systems perspective.

\section{References}

1 Berti PR, Krasevec J, FitzGerald S. A review of the effectiveness of agriculture interventions in improving nutrition outcomes. Public Health Nutr. PATH Canada, 2004; 7:599-609.

2 Ruel MT. Can food-based strategies help reduce vitamin A and iron deficiencies? A review of recent evidence. IFPRI

3 Hawkes C, Ruel MT. From Agriculture to Nutrition: Pathways Synergies and Outcomes. 2008.

4 Negin J, Remans R, Karuti S, Fanzo JC. Integrating a broader notion of food security and gender empowerment into the African Green Revolution. Food Secur; 1. 2009.

5 The World Bank. Improving nutrition through multisectoral approaches. 2013.

6 USAID multi-sectoral nutrition strategy 2014-2025.

7 Ruel MT, Alderman H. Nutrition-sensitive interventions and programmes: how can they help to accelerate progress in improving maternal and child nutrition? Lancet. 2013 Aug.

8 WHO. Global nutrition targets 2025: stunting policy brief (WHO/NMH/NHD/14.3) WHO; 2014.

9 Herforth A, Harris J. Understanding and Applying Primary Pathways and Principles. Brief \#1. Improving Nutrition through Agriculture Technical Brief Series. 2014.

10 Ruel MT, Quisumbing AR, Balagamwala M. Nutrition-sensitive agriculture: What have we learned so far? Glob Food Sec. 2018;

11 Dewey KG. Reducing stunting by improving maternal, infant and young child nutrition in regions such as South Asia. Matern Child Nutr; 12 Suppl. 2016.

12 Leroy JL, Frongillo EA. Perspective: What Does Stunting Really Mean? A Critical Review of the Evidence. Adv Nutr. 2019

13 Popkin BM, Corvalan C, Grummer-Strawn LM. Dynamics of the double burden of malnutrition and the changing nutrition reality. Lancet; 395. 2020.

14 Gibson RS, Ferguson EL. An interactive 24-hour recall for assessing the adequacy of iron and zinc intakes in developing countries. IFPRI and CIAT; 2008

15 Gibson RS. Principles of Nutritional Assessment. Oxford University Press; 2005.

16 Wirt A, Collins CE. Diet quality - what is it and does it matter? Public Health Nutr 2009;12:2473-92

17 Alkerwi A. Diet quality concept. Nutrition. 2014;30:613-8.

18 Arimond M, Deitchler M. Measuring diet quality for women of reproductive age in low- and middle-income countries: Towards new metrics for changing diets. Intake Center for Dietary Assessment/FHI 360. 2019.

19 Kim S, Haines PS, Siega-Riz AM, Popkin BM. The Diet Quality Index-International (DQI-I) provides an effective tool for cross-national comparison of diet quality as illustrated by China and the United States. J Nutr; 133. 2003.

20 Guenther PM, Casavale KO, Reedy J, Kirkpatrick SI, Hiza HAB, Kuczynski KJ, Kahle LL, Krebs-Smith SM. Update of the Healthy Eating Index: HEI-2010. J Acad Nutr Diet; 113. 2013

21 Chiuve SE, Fung TT, Rimm EB, Hu FB, McCullough ML, Wang M, Stampfer MJ, Willett WC. Alternative dietary indices both strongly predict risk of chronic disease. J Nutr; 142. 2012.

22 Working Group on Infant and Young Child Feeding Indicators. Developing and validating simple indicators of dietary quality and energy intake of infants and young children in developing countries: summary of findings from analysis of 10 data sets. FANTA Project, AED; 2006

23 Ruel MT, Deitchler M, Arimond M. Developing Simple Measures of Women's Diet Quality in Developing Countries: Overview. J Nutr; 140. 2010.

24 Hawkes C, Harris J, Gillespie S. Changing diets: Urbanization and the nutrition transition. 2017 Global Food Policy Report.
INTERNATIONAL FOOD POLICY RESEARCH INSTITUTE

$A$ world free of hunger and malnutrition

IFPRI is a CGIAR Research Center

1201 Eye Street, NW, Washington, DC 20005 USA

T. +1-202-862-5600 | F. +1-202-862-5606 | ifpri@cgiar.org | www.ifpri.org

Copyright $\odot 2020$ International Food Policy Research Institute. All rights reserved

Contact ifpri-copyright@cgiar.org for permission to republish.

Photo credits: Chris de Bode/CGIAR; Janet Hodur/A4NH.
VISIT A4NH ONLINE www.a4nh.cgiar.org

$\square$ CONTACT A4NH John McDermott A4NH Director j.mcdermott@cgiar.org
TWITTER @A4NH_CGIAR

The International Food Policy Research Institute leads A4NH's flagship on Supporting Policies, Programs, and Enabling Action through Research (SPEAR). To learn more, visit www.a4nh.org 